\title{
GAMMA/GAMMA-PRIME MICROSTRUCTURE FORMED BY PHASE SEPARATION OF GAMMA-PRIME PRECIPITATES IN A Ni-Al-Ti ALLOY
}

\author{
Minoru Doi, Daisuke Miki, Tomokazu Moritani and Takao Kozakai \\ Department of Materials Science and Engineering, Nagoya Institute of Technology \\ Gokiso-cho, Showa-ku, Nagoya 466-8555, Japan
}

Keywords: gamma-prime, Ni-base alloy, elasticity effect, precipitate coarsening, split

\begin{abstract}
The evolution of $\gamma / \gamma^{\prime}$ microstructure in elastically constrained $\mathrm{Ni}$ 8.5at.\%Al-5.4at.\%Ti alloy was investigated by means of transmission electron microscopy and the energy calculations based on a microelasticity theory. When the homogenized Ni-AlTi alloy is aged at $1213 \mathrm{~K}$, cuboidal $\gamma^{\prime}$ precipitate particles appear throughout the $\gamma$ matrix. When the $\gamma / \gamma^{\prime}$ two-phase microstructure is aged at $1023 \mathrm{~K}$ or $973 \mathrm{~K}$, the phase separation takes place in each $\gamma^{\prime}$ precipitate and a number of $\gamma$ particles newly appear in the cuboidal $\gamma^{\prime}$ precipitate. In the course of further ageing at $1023 \mathrm{~K}$ or $973 \mathrm{~K}$, the $\gamma$ particles coarsen and become plates with surfaces parallel to $\{100\}$, then some $\gamma$ plates extend along one of $\{100\}$ to become flatter, and finally reaches the $\gamma$ matrix surrounding the $\gamma^{\prime}$ precipitate. Not only the elastic energies but also the chemical free energy plays an essential role in this type of split as the driving force. The split takes place throughout the microstructure although the volume fraction is high, which results in the occurrence of the decelerated coarsening of $\gamma / \gamma^{\prime}$ two-phase microstructure in an elastically constrained $\mathrm{Ni}-\mathrm{Al}-\mathrm{Ti}$ alloy.
\end{abstract}

\section{Introduction}

Many of the properties of Ni-base superalloys are originated from the phase-separated microstructures strengthened by coherent $\gamma$ ' particles. To obtain the microstructure containing finely dispersed $\gamma$ ' precipitate particles, the phase transformation which takes place in the course of heat treatment is usually utilized. However, the desired microstructure accompanied by useful properties is, in general, obtained by interrupting the heat treatment and hence by interrupting the phase transformation. Therefore, the desired $\gamma / \gamma^{\prime}$ microstructure is usually in a metastable state from the thermodynamic point of view, and the $\gamma^{\prime}$ particles change their shape, arrangement, size, etc. to decrease their energy state towards the stable state. Furthermore, such coherent $\gamma / \gamma^{\prime}$ systems are elastically constrained more or less, and hence the elastic energy arises from the lattice mismatch between $\gamma$ and $\gamma^{\prime}$ phases. Then the effects of elastic energies, i.e. the elasticity effects, sometimes become dominant enough to control the morphology of $\gamma$ ' precipitates.

The present authors' group has been pointing out the importance of the elasticity effects on the microstructures of alloy systems containing coherent precipitates [1-8]: e.g. $\gamma / \gamma^{\prime}$ system of Ni-base alloys, $\mathrm{B} 2 / \mathrm{DO}_{3}$ system of Fe-base alloys, $\alpha-\mathrm{Ti}^{2} / \mathrm{Ti}_{3} \mathrm{Al}$ and $\mathrm{TiAl} / \mathrm{Ti}_{3} \mathrm{Al}_{5}$ systems of Ti-Al alloys, etc. Regarding the Ni-base alloys, the two-phase microstructures examined were mainly limited to those consisting of the disordered $\gamma$ matrix and the ordered $\gamma^{\prime}$ precipitate phases. However, some studies have been reported on the two-phase microstructures in which $\gamma$ particles are dispersed in the $\gamma^{\prime}$ matrix of Ni-Al-Ti alloys $[9,10]$.

In the present studies, the evolution of $\gamma / \gamma^{\prime}$ microstructure as a result of the phase separation of $\gamma^{\prime}$ precipitate particles in an elastically constrained Ni-Al-Ti alloy was investigated by means of transmission electron microscopy observations and the energy calculations based on a microelasticity theory.

\section{Experimental Procedures}

Ni-8.5at.\%Al-5.4at.\%Ti alloy was used in the present studies. An ingot prepared with vacuum induction melting was hot-forged to obtain a rod specimen of $7 \mathrm{~mm}$ in diameter and $0.7 \mathrm{~mm}$ in thickness. Each disc was solution-treated at a high temperature inside the single-phase region of $\gamma$, and was subsequently quenched into iced brine. The solution-treated disc was aged at $1213 \mathrm{~K}$ followed by quenching into iced brine, and finally was aged at $1023 \mathrm{~K}$ or $973 \mathrm{~K}$.

Thin foil specimens for transmission electron microscopy (TEM) observations were obtained by electropolishing the aged discs in an electrolyte of $10 \mathrm{vol} . \% \mathrm{H}_{2} \mathrm{SO}_{4}$ and $90 \mathrm{vol} . \% \mathrm{CH}_{3} \mathrm{OH}$ at $223 \mathrm{~K}$. The microstructure evolution caused by ageing was observed by means of TEM and was also interpreted by theoretical calculations based on a microelasticity theory from the thermodynamic point of view. Since all the TEM images were taken with the 100 superlattice reflection of ordered $\gamma^{\prime}$ phase, the bright and the dark regions correspond to $\gamma^{\prime}$ and $\gamma$ phases respectively.

\section{TEM Observations}

Precipitation of $\gamma^{\prime}$ Particles in $\gamma$ Matrix

Figure 1 illustrates the TEM image of cuboidal $\gamma^{\prime}$ precipitate particles in the $\gamma$ matrix of Ni-8.5Al-5.4Ti alloy which is solutiontreated and then aged at $1213 \mathrm{~K}$ for $2.7 \times 10^{3} \mathrm{sec}$. Due to the ageing at $1213 \mathrm{~K}$, the $\gamma$ phase formed by solid-solution treatment separates into two-phase state of $\gamma+\gamma^{\prime}$ and spherical $\gamma^{\prime}$ particles precipitate in the $\gamma$ matrix. In the course of further ageing at 1213 $\mathrm{K}$, the $\gamma$ ' precipitate particles coarsen and the shape change from sphere to cuboid takes place due to the effect of elastic energy which arises from the lattice mismatch between $\gamma$-matrix and $\gamma$ 'precipitate [11]. Furthermore, the $\gamma^{\prime}$ particles are aligned along $<100>$ directions, which is also seen in Fig. 1. The directional alignment of $\gamma^{\prime}$ particles is also a result of elastic energy and, in particular, of elastic interaction energy explained hereinafter. 


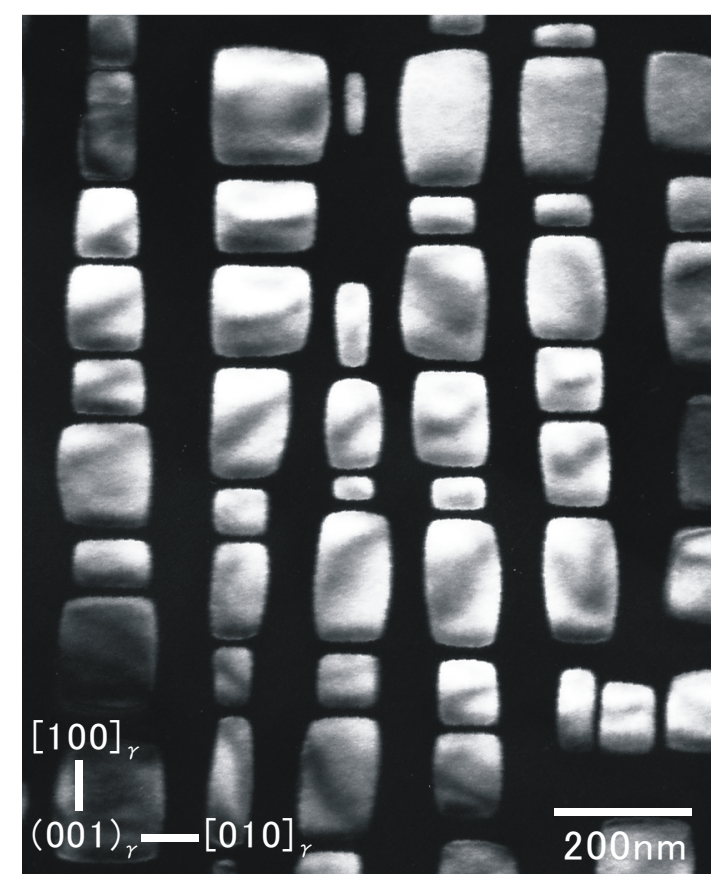

Figure 1. TEM image of cuboidal $\gamma^{\prime}$ precipitate particles aligned along $\langle 100\rangle$ directions in $\gamma$ matrix of Ni-8.5Al-5.4Ti alloy aged at $1213 \mathrm{~K}$ for $2.7 \times 10^{3}$ sec.

\section{Precipitation of $\gamma$ Particles in $\gamma$ ' Precipitate}

Figure 2 illustrates the TEM images of $\gamma / \gamma^{\prime}$ two-phase microstructures in Ni-8.5Al-5.4Ti alloy aged at $1023 \mathrm{~K}$ after ageing at $1213 \mathrm{~K}$ for $2.7 \times 10^{3} \mathrm{sec}$. Due to the ageing at $1023 \mathrm{~K}$, the phase separation of $\gamma^{\prime}$ precipitate phase takes place and a number of $\gamma$ particles newly appear here and there in the cuboidal $\gamma$ ' precipitates which act as the matrix for the phase separation. In the course of ageing at $1023 \mathrm{~K}$, the $\gamma$ particles coarsen and change their shape from cube to plate, and the $\gamma$ particles become aligned along $<100>$ directions. While further ageing at $1023 \mathrm{~K}$, the $\gamma$ plates coarsen to become flatter in cuboidal $\gamma^{\prime}$ precipitate and finally some $\gamma$ plates elongate along $\{100\}$ to reach the $\gamma$ matrix surrounding the $\gamma^{\prime}$ precipitate. This is just the split phenomenon of $\gamma^{\prime}$ precipitate particles.

Figure 3 illustrates the TEM images of $\gamma / \gamma$ ' two-phase microstructures in Ni-8.5 Al-5.4Ti alloy aged at $973 \mathrm{~K}$ after ageing at $1213 \mathrm{~K}$ for $2.7 \times 10^{3} \mathrm{sec}$. The morphological change of $\gamma$ particles which newly appear in cuboidal $\gamma^{\prime}$ precipitates is essentially the same as that caused by ageing at $1023 \mathrm{~K}$ : i.e. the split of $\gamma^{\prime}$ phase also takes place here and there throughout the two-phase microstructure. However, a minute comparison of the ageing at $973 \mathrm{~K}$ with that at $1023 \mathrm{~K}$ indicates that there exist some differences between them as follows: the number and amount of $\gamma$ particles in $\gamma^{\prime}$ precipitate are larger in the former than in the latter; the $\gamma$ particles can finely exist for longer time in the former situation.
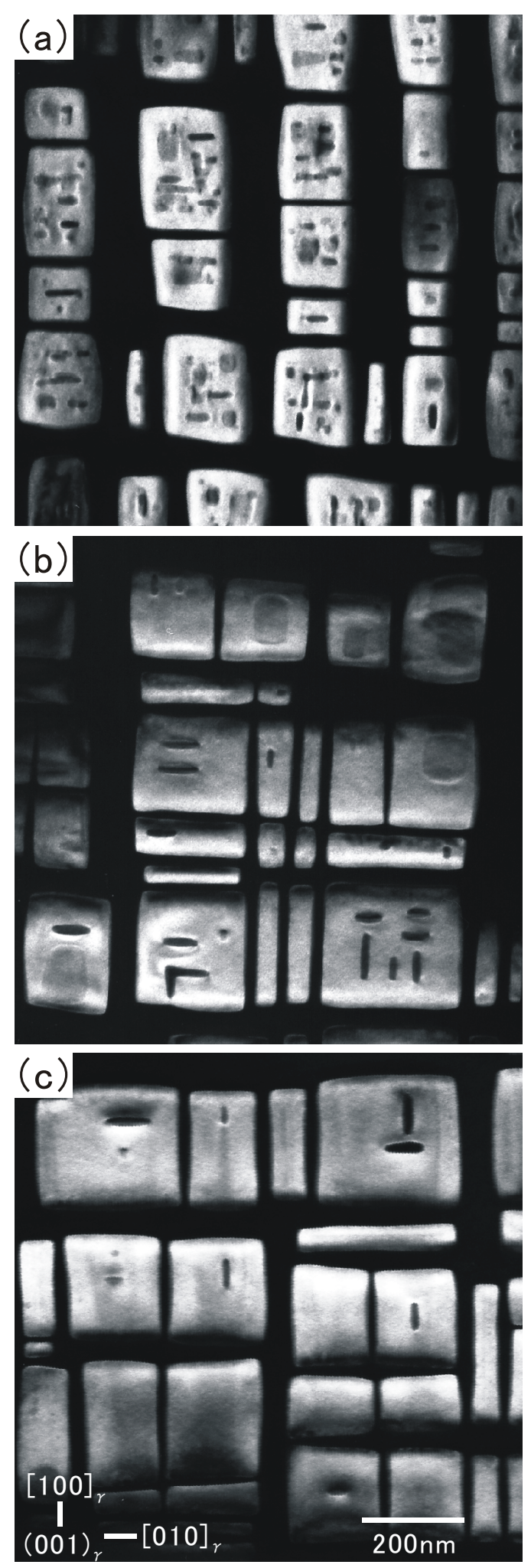

Figure 2. TEM images illustrating the precipitation of $\gamma$ particles in $\gamma^{\prime}$ precipitates in Ni-8.5Al-5.4 alloy aged at $1023 \mathrm{~K}$ for (a) $4.32 \times 10^{4} \mathrm{sec}$, (b) $1.728 \times 10^{5}$ sec and (c) $6.912 \times 10^{5} \mathrm{sec}$ after ageing at $1213 \mathrm{~K}$ for $2.7 \times 10^{3} \mathrm{sec}$. 

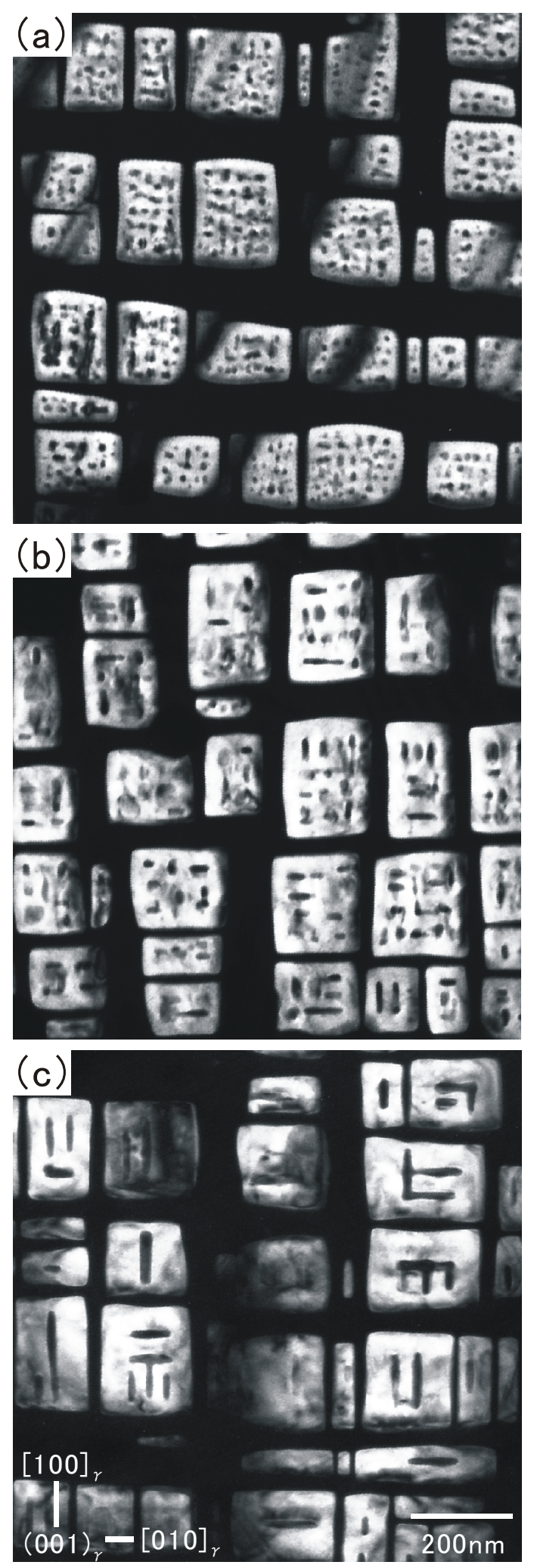

Figure 3. TEM images illustrating the precipitation of $\gamma$ particles in $\gamma^{\prime}$ precipitates in $\mathrm{Ni}$ 8.5Al-5.4 alloy aged at $973 \mathrm{~K}$ for (a) $8.64 \times 10^{4}$ sec, (b) $1.728 \times 10^{5} \mathrm{sec}$ and (c) $6.912 \times 10^{5} \mathrm{sec}$ after ageing at $1213 \mathrm{~K}$ for $2.7 \times 10^{3} \mathrm{sec}$.

\section{Theoretical Calculations}

Energy State of Coherent Particle

It goes without saying that minimizing the energy state governs the precipitate morphology from the thermodynamic point of view. Therefore, how to estimate the energy state is important for interpreting the microstructure evolution of two-phase alloys. The energy state $E_{\mathrm{TTL}}$ of a coherent particle in an infinite matrix is expressed by $[4,5]$

$$
E_{\mathrm{TTL}}=E_{\mathrm{SURF}}+E_{\mathrm{STR}}+E_{\mathrm{INT}}
$$

Here $E_{\mathrm{SURF}}$ is the surface energy of the particle (i.e. the interfacial energy between the particle and the matrix), $E_{\mathrm{STR}}$ is the elastic strain energy due to the lattice mismatch between the particle and the matrix and $E_{\mathrm{INT}}$ is the elastic interaction energy between particles.

The surface energy $E_{\mathrm{SURF}}$ is given by

$$
E_{\mathrm{SURF}}=S(p) \gamma_{\mathrm{S}}
$$

where $S(p)$ is the surface area of the particle and depends on the aspect ratio $p$ which is defined as the ratio of the length along $c$ axis to that along $a$-axis $(p \equiv c / a)$. The $\gamma_{\mathrm{S}}$ is the surface energy density.

The elastic strain energy $E_{\mathrm{STR}}$ in Eq.(1) is expressed by

$$
E_{\mathrm{STR}}=V E_{\mathrm{str}}(p)
$$

where $V$ is the volume of the particle and $E_{\text {str }}(p)$ is the elastic strain energy density of an ellipsoidal particle in an infinite and elastically anisotropic matrix. $E_{\text {str }}$ is given by Eshelby as follows [12]:

$$
E_{\mathrm{str}}=-(1 / 2) \sigma_{i j}^{\mathrm{I}}(p) e_{i j}^{\mathrm{T}^{*}}
$$

where $\sigma_{i j}^{\mathrm{I}}(p)$ is the stress inside the particle and $e_{i j}{ }^{\mathrm{T}^{*}}$ is the eigenstrain, i.e. the stress-free strain or transformation strain. If the strain is pure dilatation similar to the case of $\gamma / \gamma^{\prime}$ system of Nibase alloys, $e_{i j}{ }^{\mathrm{T} *}$ is given by

$$
\begin{aligned}
e_{i j} \mathrm{~T}^{*} & =\left(a_{\mathrm{p}}-a_{\mathrm{m}}\right) / a_{\mathrm{m}} & & \text { for } i \neq j \\
& =0 & & \text { for } i=j
\end{aligned}
$$

where $a_{\mathrm{p}}$ and $a_{\mathrm{m}}$ are the lattice constants of the particle and the matrix respectively.

When the inter-particle distance is short enough, the elastic interaction energy $E_{\mathrm{INT}}$ arises due to the overlap of the elastic strain fields around the paired particles. The $E_{\mathrm{INT}}$ between $\alpha$ and $\beta$ particles can be calculated by utilizing the following equation proposed by Yamauchi and de Fontaine [13]:

$$
E_{\mathrm{INT}}=(1 / v) \Sigma_{\mathbf{q}} F^{\alpha \beta}(\mathbf{n}) S^{\alpha}(\mathbf{q}) S^{\beta}(-\mathbf{q}) \exp (i \mathbf{q} \cdot \mathbf{L})
$$

where $v$ is the volume of the elastically anisotropic continuum containing $\alpha$ and $\beta, F^{\alpha \beta}(\mathbf{n})$ is the elastic energy coefficient in Fourier space, $\mathbf{n}$ is the unit vector along the Fourier wave vector $\mathbf{q}$, $S^{\alpha}(\mathbf{q})$ is the Fourier-space shape function of $\alpha$, and $\mathbf{L}$ is the intercentre distance between $\alpha$ and $\beta$.

When calculating the energy state of precipitate particles, we assume the individual particle as an ellipsoid of revolution to make the calculation simple. The numerical values of the lattice mismatch $e^{T^{*}}$, the surface energy density $\gamma_{\mathrm{S}}$, and the elastic constants $C_{i j}$ (for matrix) and $C_{i j}{ }^{*}$ (for precipitate) used for the present calculations are as follows: $e^{T^{*}}=0.0088 ; \gamma_{\mathrm{S}}=0.018 \mathrm{~J} / \mathrm{m}^{2}$; $C_{11}=112.4 \mathrm{GPa}, C_{12}=67.2 \mathrm{GPa}, C_{44}=56.9 \mathrm{GPa}$ for $\gamma$ ' matrix phase $\left(\mathrm{L}_{2}\right) ; C_{11}{ }^{*}=196.6 \mathrm{GPa}, C_{12}{ }^{*}=123.2 \mathrm{GPa}, C_{44}{ }^{*}=100.9 \mathrm{GPa}$ for $\gamma$ precipitate phase (A1). Here the elastic constants of $\gamma$ and $\gamma$ ' 
phases are assumed to be those of the $\gamma$ phase of pure $\mathrm{Ni}$ [14] and those of the $\gamma^{\prime}$ phase of Ni-15at.\%Al-9at.\%Ti [15] respectively. The above assumptions do not spoil the significance of the present discussions.

\section{Morphological Change of $\gamma$ Particles in $\gamma$ ' Precipitate}

Shape Change. The equilibrium particle-shape is the energetically favourable shape of a single particle, which is determined by minimizing the sum of the surface energy $E_{\mathrm{SURF}}$ and the elastic strain energy $E_{\mathrm{STR}}$. Figure 4 illustrates the energy state of a single $\gamma$ particle in the $\gamma^{\prime}$ matrix of Ni-Al-Ti alloy. The abscissa indicates the aspect ratio $p$ : the position of $p=1$ corresponds to a cube, and the right-hand side and the left-hand side of the position of $p=1$ correspond to the rod-shaped and the plate-shaped ellipsoids respectively. The ordinate is the total energy (i.e. $\left.E_{\mathrm{SURF}}+E_{\mathrm{STR}}\right)$ normalized with respect to the energy state of a cube (i.e. $p=1$ ).

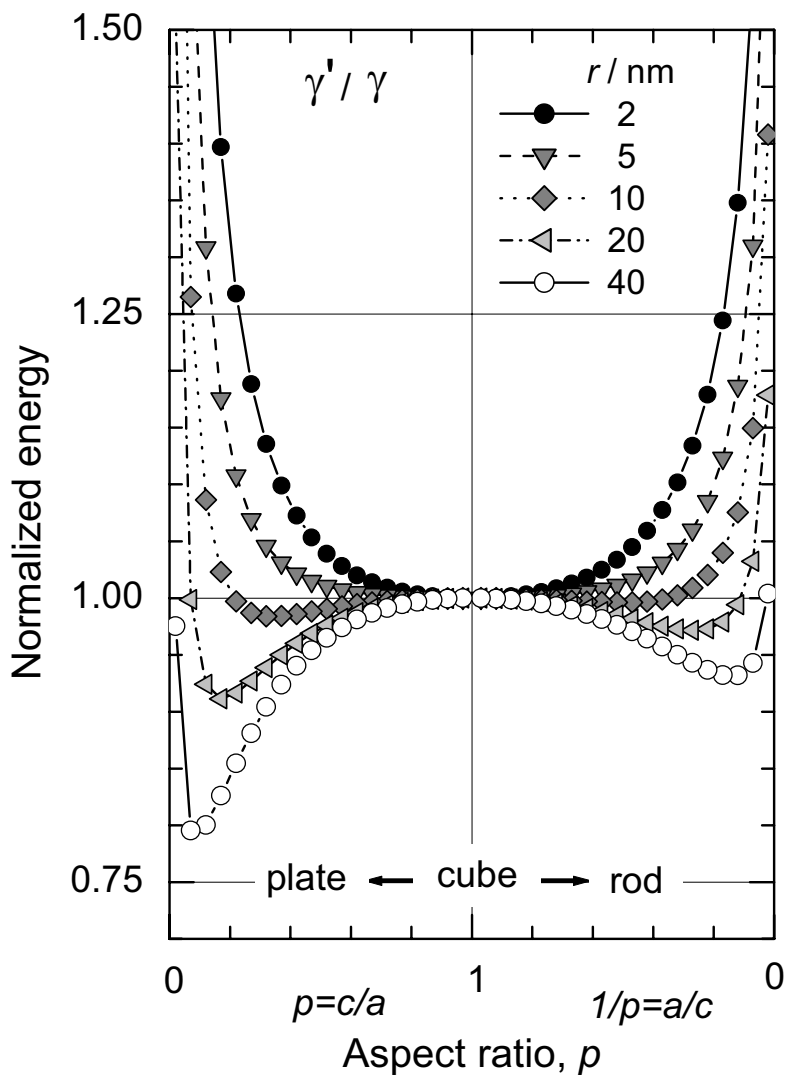

Figure 4. Energy state of a single $\gamma$ particle in the $\gamma^{\prime}$ matrix of a Ni-Al-Ti alloy as a function of particle size $r$ calculated with a microelasticity theory.

When the particle is small (e.g. $r=2 \mathrm{~nm}$ ), the energy minimum exists at $p=1$, which means that a cube is the equilibrium shape which is energetically favourable for fine particle. As the $\gamma$ particle becomes larger, two energy minima appear at the positions of larger $p$ (i.e. smaller $1 / p$ ) and smaller $p$ at a given particle size $r$. In the course of further coarsening of $\gamma$ particle, the energy minimum at the smaller $p$ side becomes the lowest. Then the energetically favourable shape becomes a plate, i.e. a flat plate is the equilibrium (stable) shape for larger particle. TEM observations clearly indicate that new $\gamma$ particles in the $\gamma$ ' precipitate (matrix) become plates in the course of coarsening as seen in Figs. 2 and 3 . The present calculation successfully reproduces the shape change of $\gamma$ particles in the actual $\mathrm{Ni}-\mathrm{Al}-\mathrm{Ti}$ alloy. This shape change is, of course, a result of the elasticity effect.

Directional Alignment. It is widely known that coherent particles are sometimes aligned along $<100>$ directions. This is due to the effect of elastic interaction energy between the particles, i.e. due to the elasticity effect. Figure 5 illustrates the elastic interaction energy between a pair of $\gamma$ particles in $\gamma$ ' matrix calculated by the method proposed by Yamauchi and de Fontaine [13]. It is clear that there exists a negative minimum when the paired particles are adjacent to each other along $<100>$ directions, as shown by the arrow in the figure. Therefore, $\gamma$ particles are aligned along $<100>$ directions in the $\gamma$ ' precipitate to create the negative energy and hence to decrease the energy state.

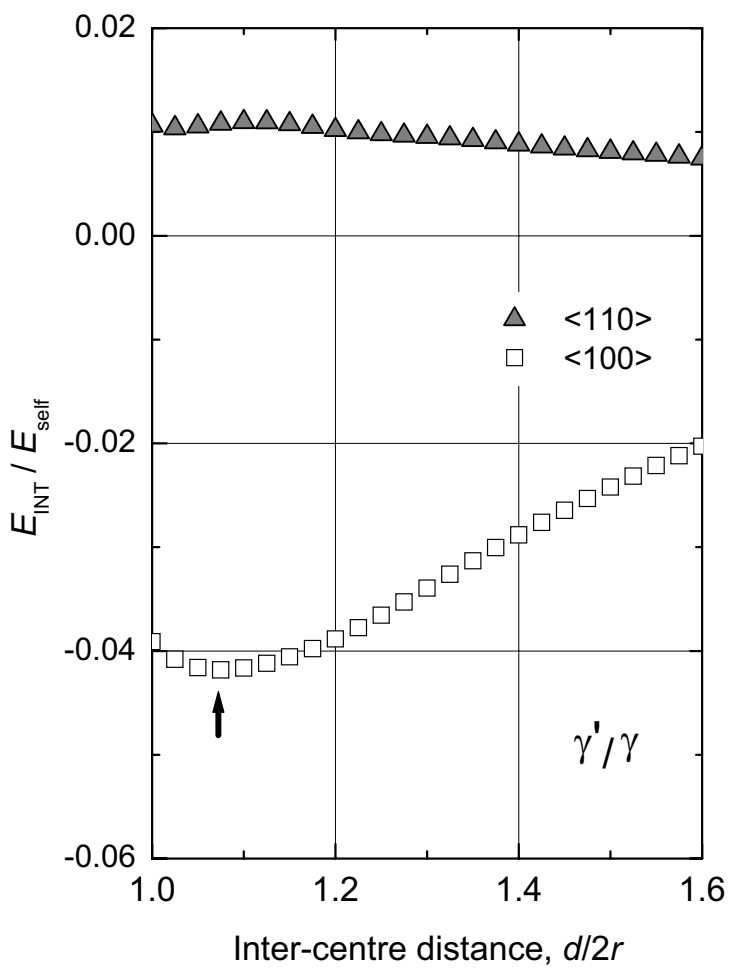

Figure 5. Elastic interaction energies between a pair of $\gamma$ precipitate particles in $\gamma^{\prime}$ precipitate (matrix). A negative minimum appears when the paired particles are adjacent to each other along $<100>$, as indicated by the arrow. 


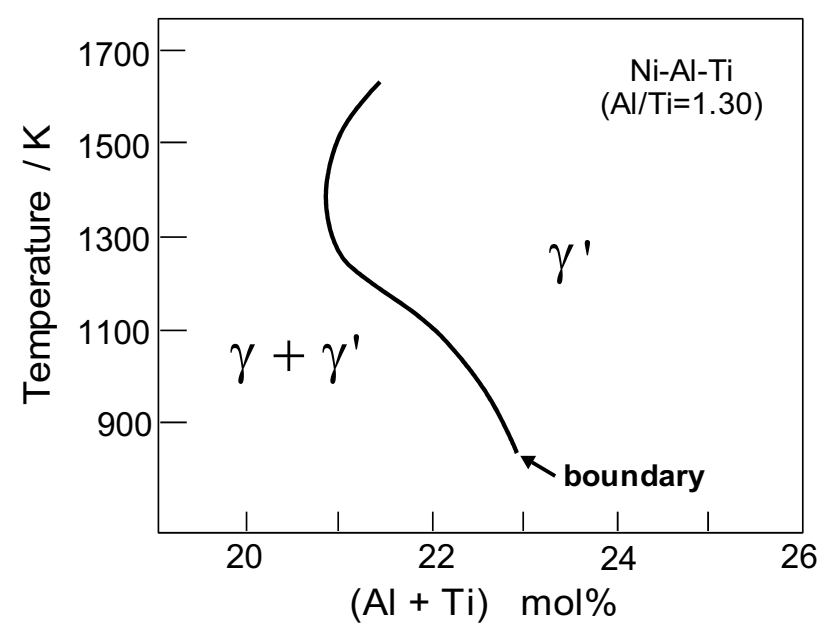

Figure 6. Boundary between the two-phase region of $\gamma^{+} \gamma^{\prime}$ and the single-phase region of $\gamma^{\prime}$ in Ni-Al-Ti ternary system (after Tian et al. [9]).

\section{Split Phenomenon and Decelerated Coarsening}

\section{$\underline{\text { Split of } \gamma^{\prime} \text { Precipitate }}$}

It is known that the split phenomenon is a result of the elastic interaction energy and has been believed to take place only when the particles are sparsely distributed in the matrix [1,4-6]. In this case having lower volume fraction, the particle must create its partner by splitting to generate the negative interaction energy to decrease the energy state. When the particle volume fraction is higher like the case of Fig. 1, however, the inter-particle distances are short enough to generate the negative interaction energy and hence the particles can decrease their energy state by simply forming the directional alignment of particles along $<100>$ directions. This means that the particles do not need to take the trouble to cause the split which seems to be more troublesome than the formation of the directional alignment by far. Nevertheless, in the actual Ni-Al-Ti alloy, the split is sometimes realised throughout the microstructure in spite of higher volume fraction, as can be seen in Figs. 2 and 3. This type of split is somewhat different from the split that has been known as a result of elastic energies only. The driving force for the split observed in the present study consists of not only the elastic energies but also the chemical free energy.

It is certain that the reason why $\gamma$ particles precipitate newly in $\gamma^{\prime}$ precipitates embedded in the $\gamma$ matrix is the difference in the volume fraction of $\gamma^{\prime}$ phase that should appear in the $\gamma / \gamma^{\prime}$ alloy system depending on the heating (ageing) temperatures. Nemoto and his collaborators [9] have studied the Ni-Al-Ti ternary phase diagram by means of TEM observations, optical microscope (OM) observations and Vickers hardness (HV) measurements. They showed the temperature dependence of the boundary between $\gamma^{\prime}$ single-phase region and $\gamma+\gamma^{\prime}$ two-phase region of $\mathrm{Ni}$ Al-Ti alloy system as indicated by the bold line in Fig. 6. This figure indicates that the two-phase region of $\gamma+\gamma$ ' expands towards the side of the lower Ni-concentration, i.e. the boundary between $\gamma+\gamma^{\prime} / \gamma^{\prime}$ shifts towards the side of $\gamma^{\prime}$ single-phase region, as the temperature decreases from about $1300 \mathrm{~K}$. The ratio of $\mathrm{Al}$ - concentration to Ti-concentration in the present study $(\mathrm{Al} / \mathrm{Ti}=1.57)$ is different to that in Fig. $6(\mathrm{Al} / \mathrm{Ti}=1.30)$. However, it is most likely that the two-phase region of $\gamma+\gamma$ ' for the case of $\mathrm{Al} / \mathrm{Ti}=1.57$ should also expand towards the side of lower Niconcentration at $1023 \mathrm{~K}$ and $973 \mathrm{~K}$ as compared with that at 1213 $\mathrm{K}$. The excess $\gamma$ phase precipitates at $1023 \mathrm{~K}$ and $973 \mathrm{~K}$ in the individual $\gamma^{\prime}$ precipitates which are already formed at $1213 \mathrm{~K}$. In other words, the chemical free energy requires the Ni-Al-Ti alloy to decrease the amount of $\gamma^{\prime}$ phase and hence new $\gamma$ particles precipitate in the individual $\gamma^{\prime}$ precipitates that act as the matrix for the $\gamma$ precipitation. The present observation of the appearance of $\gamma$ phase in $\gamma^{\prime}$ precipitates is essentially the same to that by Oblak et al. [16], but they have mentioned neither the morphological evolution of $\gamma$ phase nor the split of $\gamma^{\prime}$ precipitate.

\section{Decelerated Coarsening of $\gamma^{\prime}$ Precipitate}

Figure 7-(a) illustrates the coarsening kinetics of the $\gamma$ precipitate particles which newly appear in the $\gamma^{\prime}$ precipitates in Ni-8.5Al5.4Ti alloy aged at $1023 \mathrm{~K}$ and $973 \mathrm{~K}$. The mean particle size $\bar{r}$ in the ordinate is defined as the radius of the imaginary sphere that has the same volume to the actual particle examined. The $\gamma$ particle continues to coarsen and become a flat (thin) plate, but it reaches to the $\gamma$ matrix surrounding the $\gamma$ ' precipitate before long and the $\bar{r}$ value converges to a certain size (about $20 \mathrm{~nm}$ in Fig. 7-(a)).

Figure 7-(b) illustrates the coarsening kinetics of $\gamma^{\prime}$ precipitates, which act as the matrix for $\gamma$ precipitation, in Ni-8.5Al-5.4Ti alloy aged at $1023 \mathrm{~K}$ and $973 \mathrm{~K}$. The particle coarsening of $\gamma^{\prime}$ is practically suppressed in the course of ageing, which is most likely due to that the split phenomena occur one after another throughout the microstructure. It is expected that such a split should bring the refinement of two-phase microstructure right in the middle of particle coarsening.

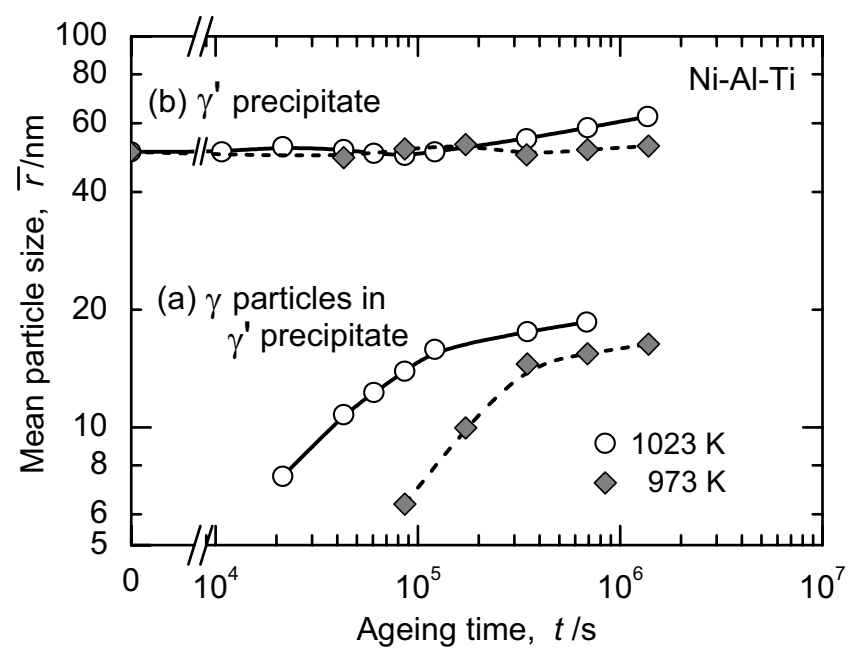

Figure 7. Coarsening kinetics of two-phase microstructure of $\gamma / \gamma^{\prime}$ of Ni-8.5Al-5.4Ti alloy aged at $1023 \mathrm{~K}$ and $973 \mathrm{~K}$ after ageing at $1213 \mathrm{~K}$ for $2.7 \times 10^{3}$ sec: (a) $\gamma$ precipitate particles in $\gamma$, precipitates; (b) $\gamma^{\prime}$ precipitate particles in $\gamma$ matrix. 


\section{Concluding Remarks}

It goes without saying that the formation and the stability of twophase microstructure containing finely dispersed second-phase particles should be very important for the practical usage of the precipitation-strengthened alloys such as Ni-base superalloys. The present studies clearly indicate that the split phenomenon really takes place even in the already developed two-phase microstructure containing finely dispersed precipitates. In this split phenomenon, not only the elastic energies but also the chemical free energy plays an essential role. Such a type of split should bring the refinement of two-phase microstructure or at least the decelerated coarsening of precipitate particles throughout the microstructure while two-phase alloys are being used. There is a good possibility of designing a stable microstructure and hence designing a new type of heat-resistant alloy by utilizing the elasticity effect and, in particular, the split phenomenon.

\section{Acknowledgements}

The authors would like to thank Mr. T. Maebashi (graduate student of Nagoya Institute of Technology) for his help in energy calculations. Part of the present studies was financially supported by a Grant-in-Aid for Scientific Research from the Japan Society for the Promotion of Science (JSPS).

\section{References}

1. M. Doi and T. Miyazaki, "The Effect of Elastic Interaction Energy on the Shape of $\gamma$ '-Precipitate in Ni-Based Alloys," Superalloys 1984, ed. by M. Gell et al. (Warrendale, PA: The Metallurgical Society of AIME, 1984), 543-552.

2. M. Doi and T. Miyazaki, "Microstructural Development under the Influence of Elastic Energy in Ni-Base Alloys Containing $\gamma$ ' Precipitates," Superalloys 1988, ed. by S. Reichman et al. (Warrendale, PA: TMS, 1988), 663-672.

3. M. Doi and T. Miyazaki, "Effect of Elastic Interaction Energy on the Distribution of Coherent Precipitate Particles in NickelBase Alloys," Superalloys 1992, ed. by S. D. Antolovich et al. (Warrendale, PA: TMS, 1992), 537-546.

4. M. Doi, "Coarsening Behaviour of Coherent Precipitates in Elastically Constrained Systems - With Particular Emphasis on Gamma-Prime Precipitates in Nickel-Base Alloys - ," Mater. Trans. JIM, 33 (1992), 637-649.

5. M. Doi, "Elasticity Effects on the Microstructure of Alloys Containing Coherent Precipitates," Prog. Mater. Sci., 40 (1996), 79-180.

6. M. Doi et al., "Transmission Electron Microscope Observations of the Splits of $\mathrm{DO}_{3}$ and B2 Precipitates in Fe-Base Alloys," Philos. Mag. Lett., 73 (1996), 331-336.

7. M. Doi et al., "Morphological Changes of the $\mathrm{Ti}_{3} \mathrm{Al}_{5}$ Phase Formed by Phase-Decomposition of TiAl Intermetallics," Mater. Sci. \& Engng. A, 329-331 (2002), 891-897.

8. M. Doi et al., "Phase-Separated Microstructures in Ti-Al Binary Alloy Systems," Proc. 10th World Conf. on Titanium (Ti2003) [in printing].

9. W.-H. Tian, T. Sano and M. Nemoto, "Precipitation of Disordered $\gamma$ Phase in an $\mathrm{L1}_{2}$ Ordered $\gamma$ ' $-\mathrm{Ni}_{3}(\mathrm{Al}, \mathrm{Ti})$ Phase," J. Jpn. Inst. Metals, 53 (1989), 1013-1021 [in Japanese].

10. T. Maebashi and M. Doi, "Coarsening Behaviours of Coherent $\gamma$ ' and $\gamma$ Precipitate Particles in Elastically Constrained Ni-Al-Ti Alloys," Mater. Sci. \& Engng. A, (2004) [in printing].
11. S. Onaka et al., "Energy Analysis with a Superspherical Shape Approximation on the Spherical to Cubical Transitions of Coherent Precipitates in Cubic Metals," Mater. Sci. \& Engng. A, 347 (2003), 42-49.

12. J. D. Eshelby, "Elastic Inclusions and Inhomogeneities," Prog. Solid Mechanics, 2 (1961), 89-140.

13. H. Yamauchi and D. de Fontaine, "Elastic Interaction of Defect Clusters with Arbitrary Strain Fields in an Anisotropic Continuum," Acta Metall., 27 (1979), 763-776.

14. T. Miyazaki, K. Nakamura and H. Mori, "Experimental and Theoretical Investigations on Morphological Changes of $\gamma$ ' Precipitates in Ni-Al Single Crystals during Uniaxial Stressannealing," J. Mater. Sci., 14 (1979), 1827-1837.

15. H. Yasuda, T. Takasugi and M. Koiwa, "Elasticity of NiBased L1 ${ }_{2}$-Type Intermetallic Compounds," Acta Metall. Mater., 40 (1992), 381-387.

16. J. M. Oblak et al., "Precipitation of $\gamma$ in the $\gamma$ ' of Nickel-Base Superalloys," Metall. Trans., 5 (1973), 1252-1255. 